\title{
A hybrid scheme for reducing peaking in high-gain observers for a class of nonlinear systems ${ }^{1}$
}

\author{
V. Andrieu ${ }^{\mathrm{a}} \quad$ C. Prieur ${ }^{\mathrm{b}} \quad$ S. Tarbouriech ${ }^{\mathrm{c}} \quad$ L. Zaccarian $^{\mathrm{c}, \mathrm{d}}$ \\ ${ }^{\text {a } L A G E P, ~ C N R S, ~ C P E, ~ U n i v e r s i t e ́ ~ L y o n ~ 1, ~ V i l l e u r b a n n e, ~ F r a n c e . ~}$ \\ ${ }^{\mathrm{b}}$ Gipsa-lab, Grenoble Campus, 11 rue des Mathématiques, BP 46, 38402 Saint Martin d'Hères Cedex, France. \\ ${ }^{\mathrm{c}} L A A S-C N R S$, Université de Toulouse, CNRS, Toulouse, France. \\ ${ }^{\mathrm{d}}$ Dipartimento di Ingegneria Industriale, University of Trento, Italy.
}

\begin{abstract}
For a family of nonlinearly interconnected second order plants, we propose a hybrid modification of the well known high-gain observer design, which leads to guaranteed asymptotic stability properties of the error dynamics, as well as exhibiting significantly reduced peaking. The hybrid modification results in suitable jumps of the observer state, which therefore corresponds to a discontinuous function of ordinary time. The jump conditions depend on the plant output and on extra states incorporated in the observer that evolve according to some suitable combination of the output error and of its integral. Our result is illustrated on a well known case study taken from the literature, and also on a planar robot with rigid links.
\end{abstract}

\section{Introduction}

The observer design problem for nonlinear dynamical systems has attracted the attention of several researchers over the last years (see the books Gauthier and Kupka (2001); Besançon (2007) and references therein). Different approaches are possible such as high-gain observers (Gauthier et al. (1992); Esfandiari and Khalil (1992); Khalil and Praly (2013) nonlinear Luenberger observers (see Andrieu and Praly (2006)), passivity (as in Ailon and Ortega (1993)) or bounds on the slope of the nonlinearity (see e.g. Arcak and Kokotovic (2001)). High-gain observers design dates back to the late 1980's (Nicosia et al. (1989); Esfandiari and Khalil (1992); Gauthier et al. (1992)) and essentially corresponds to the intuitive idea that a very strong Lyapunov decrease arising from high-gain linear output injection is capable of dominating nonlinear terms satisfying a suitable bound. Due to this intrinsic nature of the high-gain approach, these observers suffer from the so-called peaking phenomenon (Kokotovic (1992); Esfandiari and Khalil (1992); Sepulchre et al. (1997); Khalil (1999)) wherein the observer error can grow very large during the transient response. One of the interest of asymptotic observers is to use the

1 This work was supported by the ANR project LimICoS contract number 12-BS03-005-01, and by the University of Trento, grant OptHySYS. state estimation in an output feedback loop to achieve asymptotic stabilization. To protect the system from the destabilizing effect of peaking, high-gain observers have to be followed by saturation as explained in Esfandiari and Khalil (1992). This strategy has been formalized in Teel and Praly (1994) in combination with an a priori knowledge of a set of initial conditions to achieve semi global stabilization and in Kaliora et al. (2006) in combination with a norm observer. Oliveira et al. (2013, 2010) successively exploit a dwell-time control activation to avoid the peaking phenomenon.

In this paper, we focus on the state estimation problem and we don't assume that an estimation of the norm of the state is available. We revisit high-gain observer designs within the hybrid dynamical systems framework of Goebel et al. (2012, 2009) and, for a family of nonlinearly interconnected second order plants, we propose a hybrid high-gain observer having the novel feature of not exhibiting peaking when the (high) gain of the observer is increased. In particular, the observers that we propose comprise a flow dynamics (continuous-time evolution) which essentially coincides with the original continuoustime high-gain observer of Esfandiari and Khalil (1992); Nicosia et al. (1989); Khalil (1999), augmented with a suitable resetting rule enforced on the observer state. Such a scheme ensures that trajectories approaching a region of the state space where peaking occurs, are projected in another region where peaking is absent. We 
show that the Lyapunov function establishing exponential stability of the error system does not increase, thus preserving the asymptotic state estimation property of the observer. Alternative hybrid observation schemes have been presented in, e.g., Raff and Allgöwer (2008); Paesa et al. (2012) and reference therein, which don't specifically focus on peaking reduction.

Preliminary results in the directions of this paper have been presented in Prieur et al. (2012). Here, as compared to those preliminary results, we extend our theory from a single second-order plant to a family of nonlinearly interconnected second-order plant, which allows us to address the case of joint speed estimation in $n$ degrees of freedom Euler-Lagrange systems. Moreover, we propose different resetting laws and jump and flow sets, to allow for a suitable adaptation of the results to the $2 n$-dimensional case. Another observer approach to avoid overshoot in the case of planar multidimensional systems is the reduced order observer. More precisely, this framework presented in (Besançon, 2000, Lemma 3.1) computes a reduced order observer for any system for which a high-gain observer has been computed and thus leads to a reduction of the overshoot. In the context of planar observer (that is $n=1$ in (1) below), the obtained observer is scalar and has a decreasing state estimation error. However the estimate has a feedthrough term from the measurement output and thus is more sensitive to measurement noise. The hybrid observer that is designed in the present paper can be seen as a tradeoff between these two techniques. Recently in Astolfi and Praly (2013) another approach to avoid peaking has been investigated. It employs a projection approach and requires the knowledge of a convex set in which the state trajectory remains. This is not required in our approach.

The paper is organized as follows. Section 2 is dedicated to revise the high-gain observer by introducing suitable resetting laws for the state of the observer in order to reduce the peaking. Section 3 deals with an application of the results to a robotic example.

Notation: Throughout the paper, notation is standard. $|\cdot|$ stands for the Euclidean norm. For any vector $x$ (resp. matrix $A$ ), $x^{T}$ (resp. matrix $A^{T}$ ) denotes its transpose.

\section{A hybrid observer with reduced peaking}

Before considering a hybrid observer in Subsection 2.2 below, let us first define the class of nonlinear planar systems and the associated high-gain observer. The peaking phenomenon is also briefly discussed and illustrated.

\subsection{High-gain observer design and Lyapunov properties}

Following the standard approach in, e.g., Esfandiari and Khalil (1992), we consider a set of $n$ second order nonlin- ear systems whose state is denoted by $x=\left(x_{1}, \ldots, x_{n}\right) \in$ $\mathbb{R}^{2 n}$ and $x_{i}=\left(p_{i}, v_{i}\right) \in \mathbb{R}^{2}$, where each substate $x_{i}$ obeys the following second order nonlinear dynamics with nonlinear couplings arising from the terms $\psi_{i}$ and $\phi_{i}$ :

$$
\begin{aligned}
& \dot{p}_{i}=v_{i}+\psi_{i}(y), \dot{v}_{i}=\phi_{i}(x, u) \\
& y_{i}=p_{i}
\end{aligned}
$$

for all $i=1, \ldots, n$, where $y=\left(y_{1}, \ldots, y_{n}\right) \in \mathbb{R}^{n}$ is the measurement output, $u \in \mathbb{R}^{p}$ is a known control input that is piecewise continuous 2 and $\psi_{i}$ and $\phi_{i}, i=1, \ldots, n$ are known nonlinearities establishing a nonlinear coupling among the $n$ planar systems. For example, in the robot example of Section 3, these couplings are caused by strong nonlinear coupling effects between the two joints.

Remark 1 Note that in Esfandiari and Khalil (1992) an output feedback is designed assuming that the nonlinearities $\phi_{i}$ are uncertain. In this paper, since we address the asymptotic observer design problem we consider the case in which dynamics are perfectly known (as Gauthier and Kupka (2001) or Besançon (2007) for instance). $\lrcorner$

High-gain observers are used to provide an estimate $\hat{x}$ of the state $x$. If the following Lipschitz-like condition holds for a suitable constant $L_{\delta}>0$ :

$\left|\phi_{i}(x, u)-\phi_{i}(\hat{x}, u)\right| \leq L_{\delta}|x-\hat{x}|, \forall i=1, \ldots, n$

for all $x$ and $\hat{x}$ in $\mathbb{R}^{2 n}, u$ in $\mathbb{R}^{p}$, then a high-gain observer can be designed to ensure asymptotic stability of the origin of the feedback system from the observed state.

Remark 2 Lipschitz condition (2) is standard in the context of high-gain observer designs. Note however that it has been relaxed in Lei et al. (2005) allowing for a strictly increasing high-gain parameter. We do not follow this route since this approach may lead to undesirable behavior in the context of disturbed measurements. Moreover, this Lipschitz condition has been also slightly relaxed in Andrieu et al. (2009) employing homogeneous tools. Possible extensions of our tools in these directions are object of future work.

For system (1) we can design the following high-gain observer:

$$
\dot{\hat{x}}_{i}=\left[\begin{array}{c}
\dot{\hat{p}}_{i} \\
\dot{\hat{v}}_{i}
\end{array}\right]=\left[\begin{array}{c}
\hat{v}_{i}+\psi_{i}(y)+\ell k_{1} e_{p i} \\
\phi_{i}(\hat{x}, u)+\ell^{2} k_{2} e_{p i}
\end{array}\right]=: f_{i}\left(\hat{x}, e_{p i}, u\right),
$$

$i=1, \ldots, n$, where $e_{p i}=y_{i}-\hat{p}_{i}$ is the $i$-th output error, $k_{1}$ and $k_{2}$ are any pair of positive scalars, and $\ell$ is

2 The assumption on piecewise continuity of input $u$ could be relaxed but special care has to be paid to the hybridization of the corresponding function of time (see, e.g., Sanfelice (2014) for details). 
the "high" gain of the high-gain observer. The following dynamics can be easily derived from (1), (3) if one defines the scaled error coordinates $e_{i}:=\left(e_{p i}, e_{v i}\right):=\left(p_{i}-\right.$ $\left.\hat{p}_{i}, \ell^{-1}\left(v_{i}-\hat{v}_{i}\right)\right)$ :

$$
\begin{aligned}
\dot{e}_{i} & =\ell\left[\begin{array}{ll}
-k_{1} & 1 \\
-k_{2} & 0
\end{array}\right] e_{i}+\left[\begin{array}{c}
0 \\
\frac{\phi_{i}(x, u)-\phi_{i}(\hat{x}, u)}{\ell}
\end{array}\right] \\
& =: \ell A_{e} e_{i}+\left[\begin{array}{c}
0 \\
\delta_{i}(x, \hat{x}, u)
\end{array}\right], \quad i=1, \ldots, n
\end{aligned}
$$

which reveals the potential for the high-gain $\ell \gg 1$ to dominate over each nonlinear term $\delta_{i}, i=1, \ldots, n$. In particular, from (2), if $\ell \geq 1$, we obtain

$$
\left|\delta_{i}(x, \hat{x}, u)\right| \leq \frac{L_{\delta}}{\ell}|x-\hat{x}| \leq L_{\delta}|e|
$$

A possible way to characterize the set of sufficiently highgains $\ell$ ensuring exponential stability of the error dynamics (4) is given in the next proposition, which is given here for completeness and will be useful for our results.

Proposition 1 Consider plant (1) satisfying (2) and observer (3), and pick any matrix $P=P^{T}>0$ such that $A_{e}^{T} P+P A_{e}<0$. Then, for each $\vartheta>0$, there exists a high-gain parameter $\ell \geq 1$ such that

$$
\left[\begin{array}{cc}
\ell\left(A_{e}^{T} P+P A_{e}\right)+n \vartheta L_{\delta}^{2} I_{2} & P\left[\begin{array}{l}
0 \\
1
\end{array}\right] \\
\left(P\left[\begin{array}{l}
0 \\
1
\end{array}\right]\right)^{T} & -\vartheta
\end{array}\right]<0 .
$$

Moreover, with such a selection of the high-gain $\ell$, the origin is globally exponentially stable for the error $d y$ namics (4), as established by the Lyapunov function

$$
e \mapsto V(e):=\sum_{i=1}^{n} e_{i}^{T} P e_{i}=e^{T}\left(P \otimes I_{n}\right) e
$$

where $e=\left(e_{1}, \ldots, e_{n}\right)$ and $I_{n} \in \mathbb{R}^{n \times n}$ is the identity matrix.

Remark 3 Before proving Proposition 1, let us note that in its context, and recalling the definition of the scaled error, Lemma 3.1 of Besançon (2000) applies and leads to the following reduced order observer $\hat{x}_{i}=\left[\begin{array}{l}y_{i} \\ \hat{v}_{i}\end{array}\right]$ for $i=1, \ldots, n$ where $\hat{v}_{i}=w_{i}+p_{11}^{-1} p_{12} p_{i}$ and $w_{i}$ has the following dynamics $\dot{w}_{i}=\phi_{i}(\hat{v}, u)+\frac{p_{12}}{p_{11} \ell} \hat{v}_{i}$ where $P=\left[\begin{array}{cc}p_{11} & p_{12} \\ \star & p_{22}\end{array}\right]$. With this observer, the Lyapunov function of the error system is $\sum_{i}\left(v_{i}-\hat{v}_{i}\right)^{2}$, which has balanced coordinates. This is not the case for Lyapunov function (7) in the original error coordinates. Therefore the reduced order observer prevents in general peaking, but its estimate $\hat{v}_{i}$ depends directly on $p_{i}$ and is therefore expected to be more sensitive to measurement noise. $\lrcorner$
Proof of Proposition 1. Regarding exponential stability of (4), we show next that $V$ is a Lyapunov function for dynamics (4). To this aim, from (5) we get

$$
|\delta(x, \hat{x}, u)|:=\left|\left[\begin{array}{c}
\delta_{1}(x, \hat{x}, u) \\
\vdots \\
\delta_{n}(x, \hat{x}, u)
\end{array}\right]\right| \leq \sqrt{n} L_{\delta}|e| .
$$

Then, from (8) and for any $\vartheta \geq 0$, we can write for all $x$ and $\hat{x}$ in $\mathbb{R}^{2 n}$,

$$
\begin{gathered}
{\left[\begin{array}{c}
e \\
\delta(x, \hat{x}, u)
\end{array}\right]^{T}\left[\begin{array}{cc}
-\vartheta n L_{\delta}^{2} I_{2 n} & 0 \\
0 & \vartheta I_{n}
\end{array}\right]\left[\begin{array}{c}
e \\
\delta(x, \hat{x}, u)
\end{array}\right]} \\
\quad=\vartheta\left(\delta(x, \hat{x}, u)^{T} \delta(x, \hat{x}, u)-n L_{\delta}^{2} e^{T} e\right) \leq 0 .
\end{gathered}
$$

Now let us rewrite dynamics (4) as follows:

$$
\dot{e}=\ell\left(A_{e} \otimes I_{n}\right) e+\left(\left[\begin{array}{l}
0 \\
1
\end{array}\right] \otimes I_{n}\right) \delta(x, \hat{x}, u)
$$

and note that using the well known relation $(A \otimes B)(C \otimes$ $D)=A C \otimes B D$, the derivative of the function $V$ in $(7)$ along dynamics (10) can be bounded as follows, where we also use (9):

$$
\begin{aligned}
& \dot{V}=2 e^{T}\left(P \otimes I_{n}\right)\left(\ell\left(A_{e} \otimes I_{n}\right) e+\left(\left[\begin{array}{l}
0 \\
1
\end{array}\right] \otimes I_{n}\right) \delta(x, \hat{x}, u)\right) \\
& =e^{T} 2 \ell\left(P A_{e} \otimes I_{n}\right) e+e^{T} 2\left(P\left[\begin{array}{l}
0 \\
1
\end{array}\right] \otimes I_{n}\right) \delta(x, \hat{x}, u) \\
& \leq\left[\begin{array}{l}
e \\
\delta
\end{array}\right]^{T}\left[\begin{array}{cc}
\left(\ell\left(P A_{e}+A_{e}^{T} P\right)+\vartheta n L_{\delta}^{2} I_{2}\right) \otimes I_{n} & \star \\
\left(P\left[\begin{array}{l}
0 \\
1
\end{array}\right] \otimes I_{n}\right)^{T} & -\vartheta \otimes I_{n}
\end{array}\right]\left[\begin{array}{l}
e \\
\delta
\end{array}\right], \\
& <-\epsilon_{V}\left(|e|^{2}+|\delta(x, \hat{x}, u)|^{2}\right) \text {, }
\end{aligned}
$$

where $\star$ denotes symmetric entries, $\delta$ is a shortcut notation for $\delta(x, \hat{x}, u)$ and where the last inequality holds for some small enough $\epsilon_{V}>0$ from combining the strict inequality in (6) with standard properties of the Kronecker product. Since $V$ is quadratic in $e$, with (Khalil 2002, Thm 4.10), this implies exponential stability of the origin for the error dynamics (4).

Remark 4 Since $A_{e}^{T} P+P A_{e}<0$, there exist $\vartheta>0$ and $\ell \geq 1$ such that (6) holds. It should be noted that (6) is more conservative than the worst case necessary condition for obtaining $\dot{V}<0$ in (11). This, in light of bound (5) can be written as $\sum_{i=1}^{n} \ell e_{i}^{T}\left(P A_{e}+A_{e}^{T} P\right) e_{i}+$ $2 \sqrt{n} L_{\delta}\left|e_{i}^{T} P\left[\begin{array}{l}0 \\ 1\end{array}\right]\right||e|<0$ for all $e \neq 0$. The important advantage of (6) with respect to the above necessary condition is that it is quasi-convex with respect to the unknown variables $P>0, \ell, \vartheta$ and thus defines a quasiconvex domain. It is thus natural to compute the optimal solution to

$$
\min _{P>0, \ell, \vartheta} \ell, \text { subject to }(6)
$$

so that one then implements the smallest possible gain guaranteeing global exponential stability (GES) of the 
origin for the error dynamics. The degree of conservativeness of (6) with respect to the above mentioned necessary condition is easily understood from the proof of Proposition 1, which is based on an application of the S-procedure (a necessary and sufficient condition) applied to bound (8). Therefore (6) is necessary under the worst case condition (8), which is in general more conservative than (5), even though it is equivalent to (5) in the case $n=1$. For $n>1$, the increased conservativeness allows to formulate the quasi-convex optimization problem (12).

Example 1 To motivate our work, we consider the following example, inspired by (Esfandiari and Khalil, 1992. Example 3) (function $\phi(\cdot, \cdot)$ is perfectly known here while it is uncertain in Esfandiari and Khalil (1992)) and corresponding to the case $n=1$ so that the state $x=x_{1}=\left(p_{1}, v_{1}\right)=(p, v)$ is two-dimensional:

$$
\dot{p}=v, \dot{v}=a_{1} \sin (p)+b_{1} u
$$

where $a_{1}=1.4$ and $b_{1}=0.8$ are two constant values. As explained in Khalil (1999), this example illustrates the peaking phenomenon, and shows how high gain can destabilize the closed-loop system (see also (Sepulchre et al. 1997, Sections 1.3.2 and 4.5) where the consequences of peaking are studied). The high-gain observer (3) is designed with the parameter $\ell$ satisfying (6) based on the following condition, which is easily proven to hold with $L_{\delta}=\frac{3}{2}$ :

$$
\begin{aligned}
|\delta(x, \hat{x}, u)| & =|\phi(x, u)-\phi(\hat{x}, u)|=\mid a_{1}(\sin (p)-\sin (\hat{p}) \mid, \\
& \leq a_{1}|p-\hat{p}| \leq \frac{3}{2}|p-\hat{p}| .
\end{aligned}
$$

Using $k_{1}=k_{2}=1$, we may check that the assumption of Proposition 1 is satisfied with $P=\left[\begin{array}{cc}1 & -0.5 \\ -0.5 & 1\end{array}\right]$ and that (6) holds with $\vartheta=2$ and $\ell=20$ (the high gain $\ell=20$ is also used ${ }^{3}$ in Esfandiari and Khalil (1992)). Therefore the conclusion of Proposition 1 holds and the scaled error system (4) is exponentially stable. Note that exponential stability of (4) is guaranteed in this example by using the quadratic Lyapunov function $V(e)=e^{T} P e$.

To illustrate the peaking phenomenon on simulations, let us consider (as in Esfandiari and Khalil (1992)) the initial condition $(p(0), v(0), \hat{p}(0), \hat{v}(0))=(1,0,0,0)$. The phase portraits of $\left(e_{p}, e_{v}\right)$ and $(p-\hat{p}, v-\hat{v})=\left(e_{p}, \ell e_{v}\right)$ are given in Figure 1. We may check on this simulation that the choice of the high-gain $\ell=20$ induces a peaking phenomenon for the error coordinates $x-\hat{x}$ (right plot) but not for the scaled error coordinates $e$ (left plot).

3 It corresponds to the value $\varepsilon=0.05$ using the notation of Esfandiari and Khalil (1992). It is also interesting to observe that the quasi-convex GEVP in (12) leads to the less conservative estimate $\ell^{*}=3.94$.
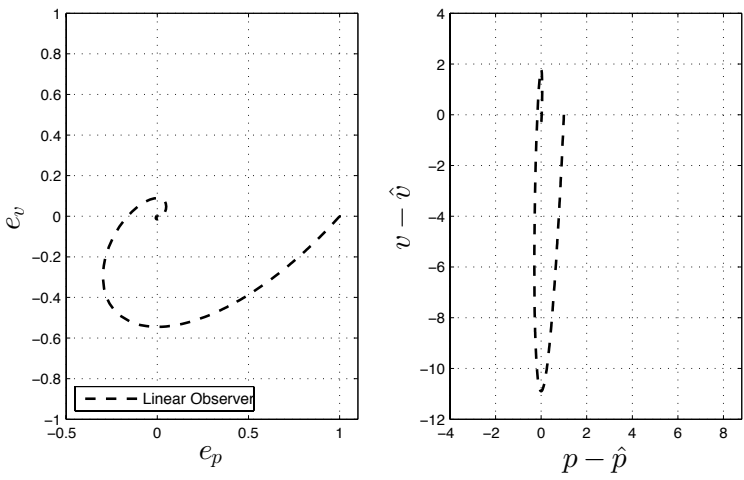

Figure 1. Phase portrait of the coordinates $\left(e_{p}, e_{v}\right)$ (left) and of $(p-\hat{p}, v-\hat{v})$ (right).

The undesirable peaking phenomenon illustrated by Example 1 (see Figure 1) can be understood well in terms of the trend of the norm of the estimation error $|x-\hat{x}|=$ $\left|\left[\begin{array}{c}e_{p i} \\ e_{v i}\end{array}\right]\right|$. A coarse understanding of the trend of this error can be gained by neglecting the nonlinear terms in the error dynamics (4), because these are dominated by the high-gain linear dynamics (see Proposition 1). Then, neglecting those nonlinear terms, one obtains:

$$
\frac{d}{d t}|x-\hat{x}|^{2}=-2 \ell\left(k_{1} e_{p i}^{2}+\left(\ell^{2} k_{2}-1\right) e_{p i} e_{v i}\right),
$$

which indicates that the flow dynamics (4) naturally enforces non increase of the norm of the estimation error in the quadrants $e_{p i} e_{v i} \geq 0$, whereas the peaking effect (increase of $|x-\hat{x}|$ ) arises in the second and fourth quadrants where $e_{p i} e_{v i} \leq 0$. Thus one would expect that a reasonable thing to do when $e$ belongs to the second (or fourth) quadrant is to change the sign of $e_{p i}$, thus jumping on the opposite side of the vertical axis so that the size of $e_{v i}$ will stop increasing (see also Figure 2). The following result substantiates this intuition.

Lemma 1 Consider the matrices in (4) and assume that (6) holds for some $P, \vartheta$. Given any e satisfying $e_{p k} e_{v k} \leq 0$ for some $k \in\{1, \ldots, n\}$, define $e^{+}$as the vector with the following components:

$e_{i}^{+}= \begin{cases}e_{i}, & \text { if } i \in\{1, \ldots, n\} \backslash\{k\} \\ \left(-\alpha e_{p i}, e_{v i}\right), & \text { if } i=k,\end{cases}$

where $\alpha \in[0,1]$ is a real number. Then the function $V$ defined in ( 7$)$ satisfies $V\left(e^{+}\right) \leq V(e)$.

Proof. Since inequality (6) implies $A_{e}^{T} P+P A_{e}<0$, then, from the structure of $P$ and $A_{e}$ we have that the elements of $P:=\left[\begin{array}{cc}p_{12} & p_{12} \\ p_{12} & p_{2}\end{array}\right]$ satisfy: $A_{e}^{T} P+P A_{e}=\left[\begin{array}{cc}\# & \# \\ \# & 2 p_{12}\end{array}\right]<0$, where \# denotes "don't care" entries. Since the diagonal terms must be negative, we have $p_{12}<0$. Consider now 
the equation

$$
\left(e_{k}^{+}\right)^{T} P e_{k}^{+}-e_{k}^{T} P e_{k}=e_{k}^{T}\left[\begin{array}{cc}
\left(\alpha^{2}-1\right) p_{1} & \star \\
-(1+\alpha) p_{12} & 0
\end{array}\right] e_{k}
$$

where $\star$ denotes symmetric terms. The term $(1,1)$ in (15) is negative and the term $(1,2)$ is positive. Therefore as long as $e_{p k} e_{v k} \leq 0$, it holds $\left(e_{k}^{+}\right)^{T} P e_{k}^{+}-e_{k}^{T} P e_{k} \leq 0$ which concludes the proof of Lemma 1.

\subsection{Hybrid observer equations and properties of solu- tions}

In this section we inherit the continuous-time dynamics (3) of the high-gain observer and introduce suitable resetting laws for the estimate $\hat{x}$ in such a way to reduce the peaking along the intuitions provided by Lemma 1 . This is formally done by considering the following hybrid system and by denoting the overall state $\xi=(x, \hat{x}, \zeta, \eta) \in \mathbb{R}^{2 n} \times \mathbb{R}^{2 n} \times \mathbb{R}^{n} \times \mathbb{R}^{n}:$

$$
\begin{aligned}
& \xi \in \mathcal{C},\left\{\begin{array}{l}
\dot{\hat{x}}_{i}=f_{i}\left(\hat{x}, e_{p i}, u\right) \\
\dot{\zeta}_{i}=0 \\
\dot{\eta}_{i}=\left(y_{i}-\hat{y}_{i}\right)^{2},
\end{array}\right. \\
& \xi \in \mathcal{D}_{i}^{p},\left\{\begin{array}{l}
\hat{x}_{i}^{+}=\hat{x}_{i} \\
\zeta_{i}^{+}=\left(y_{i}-\hat{y}_{i}\right)^{2} \\
\eta_{i}^{+}=0, \\
\hat{x}_{i}^{+}=\left[\begin{array}{l}
y_{i}-\alpha\left(\hat{p}_{i}-y_{i}\right) \\
\hat{v}_{i}
\end{array}\right] \\
\zeta_{i}^{+}=\alpha^{2}\left(y_{i}-\hat{y}_{i}\right)^{2} \\
\eta_{i}^{+}=0,
\end{array}\right.
\end{aligned}
$$

where $i=1, \ldots, n$ and in the jump rules the unspecified states remain constant across jumps and $\alpha \in[0,1]$. The flow and jump sets $\mathcal{C}$ and $\mathcal{D}$ are defined as:

$$
\begin{aligned}
& \mathcal{C}:=\left\{\xi \in \mathbb{R}^{6 n}:-\Delta_{m} \leq e_{p i}^{2}-\zeta_{i}+2 \ell k_{1} \eta_{i} \leq \Delta_{p}, \forall i\right\} \\
& \mathcal{D}:=\bigcup_{i=1}^{n} \mathcal{D}_{i}^{p} \cup \mathcal{D}_{i}^{m} \\
& \mathcal{D}_{i}^{p}:=\left\{\xi \in \mathbb{R}^{6 n}: e_{p i}^{2}-\zeta_{i}+2 \ell k_{1} \eta_{i} \geq \Delta_{p}\right\} \\
& \mathcal{D}_{i}^{m}:=\left\{\xi \in \mathbb{R}^{6 n}: e_{p i}^{2}-\zeta_{i}+2 \ell k_{1} \eta_{i} \leq-\Delta_{m}\right\}
\end{aligned}
$$

where $\zeta=\left(\zeta_{1}, \ldots, \zeta_{n}\right), \eta=\left(\eta_{1}, \ldots, \eta_{n}\right)$ and $\Delta_{p}>0$, $\Delta_{m}>0$. Using the notation in Goebel et al. (2012, 2009), the hybrid dynamics should be interpreted taking the jump set as the union of all the jump sets $\mathcal{D}_{i}^{m}, \mathcal{D}_{i}^{p}$ (as specified in (16d) and a set-valued jump map corresponding to the outer semicontinuous hull (see (Rockafellar and Wets, 1998, pp. 154-155)) of the $2 n$ jump maps in (16b)-(16c), which may be written as follows:

$$
G(\xi):=\left(\bigcup_{i: \xi \in \mathcal{D}_{i}^{p}} g_{i}^{p}(\xi)\right) \cup\left(\bigcup_{i: \xi \in \mathcal{D}_{i}^{m}} g_{i}^{m}(\xi)\right),
$$

where $g_{i}^{p}$ and $g_{i}^{m}, i=1, \ldots, n$, denote the functions specified in (16b) and (16c), respectively, augmented with trivial identity functions for the remaining components of the states (see also Phillips and Sanfelice (2015) for this type of jump map constructions with outer semicontinuous hulls). Then when any of the $i$-th jumps in $(16 b),(16 c)$ is enabled, the solution jumps following that specific jump rule for component $i$ while the other components remain unchanged across the jump. Then, the overall hybrid closed loop between plant (1) and observer (16) is understood as the combination of the flow and jump dynamics in (16) with the flow dynamics in (1) and the trivial (omitted) jump dynamics associated to the states that remain constant among jumps. For example, one always has $x^{+}=x$, resembling the fact that the (continuous-time) plant state $x$ never jumps. Note also that the proposed jump rules all imply $\zeta_{i}^{+}=\left(e_{p i}^{+}\right)^{2}$ and $\eta_{i}^{+}=0$, so that $\left(e_{p i}^{+}\right)^{2}-\zeta_{i}^{+}+\ell k_{1} \eta_{i}^{+}=0$ so that $\xi^{+} \notin \mathcal{D}_{i}^{p} \cup \mathcal{D}_{i}^{m}$.

The following proposition establishes well behavedness of the proposed hybrid observer in terms of number of jumps. In particular, since we are interested in continuous-time observation laws, the proposition ensures that all solutions satisfy an average dwell-time condition so that no Zeno solutions may occur and all maximal solutions are complete and have unbounded time domain in the ordinary time direction. Its proof is an extension of the proof of our main result and is therefore given at the end of the next section.

Proposition 2 Consider the hybrid closed loop between plant (1) and observer (16). If (6) holds for some $P$ and $\vartheta$, then all maximal solutions are complete and each solution has a hybrid domain satisfying an average dwell-time condition.

Remark 5 Hybrid observer (16) produces a hybrid estimate $\hat{x}$ of the continuous plant state $x$. By Proposition 2 , this estimate $\hat{x}$, whose domain dom $\hat{x}$ is a hybrid time domain, can be projected on the ordinary time axis $t$, thereby obtaining a discontinuous function of time whose value at the jump instants may be selected in multiple ways depending on the type of projection method (for example imposing that the function be left- or right-continuous). Note that the speed estimates $\hat{v}_{i}, i=1, \ldots, n$ are continuous functions of the ordinary time, indeed from (16b) and (16c) it is clear that $\hat{v}_{i}$ does not change across jumps. However, the position estimates $\hat{p}_{i}$ do experience discontinuities across jumps occurring from $\mathcal{D}_{i}^{m}$ (see $(16 \mathrm{c})$ ), therefore these estimates are discontinuous functions of the ordinary time t. In our preliminary work (Prieur et al., 2012, §IV), we 
proposed an extension of observer (16) that makes suitable use of a hybrid logic state $q$ toggling between -1 and 1 to obtain a continuous estimate of the position. We avoid repeating here the same type of generalization that can be obtained by following the steps in (Prieur et al. 2012, §IV). We emphasize, in addition, that the toggling therein suggested is such that when $q=-1$ one obtains a direct feedthrough term from the output $y$ to the state estimate $\hat{x}$ (see (Prieur et al., 2012, eqn (15))). Due to this reason, and since we insist here on the advantages arising from the use of a strictly proper observer as compared to an observer with feedthrough terms (see the discussion at the end of Remark 3), we promote the observer dynamics (16), which is an enhanced generalization of the preliminary observation law proposed in (Prieur et al. 2012, §III).

\subsection{Proof of convergence of the error dynamics}

In hybrid observer (16), for each $i \in\{1, \ldots, n\}$, as long as $\xi \in \mathcal{D}_{i}^{p}$, we implement the resetting law suggested by Lemma 1 and corresponding to (14). Indeed, note that the first equation in (16c) implies $e_{p i}^{+}=y_{i}^{+}-\hat{p}_{i}^{+}=-\alpha e_{p i}$.

In addition, to suitably define the jump and flow sets, hybrid observer (16) implements two extra states $\zeta_{i}$ and $\eta_{i}$. The state $\zeta_{i}$ remains constant during flows and, at each jump time, jumps to the value of the $i$-th squared output error immediately after the jump, namely $\left(e_{p i}^{+}\right)^{2}$ (this fact can be easily seen by inspecting (16b) and $(16 \mathrm{c}))$. This means that $\zeta_{i}$ acts like a sample and hold of the value of $e_{p i}^{2}$ immediately after the latest jump. The state $\eta_{i}$, instead, is reset at zero at each jump time and then integrates the $i$-th squared output error $e_{p i}^{2}$ during flows. Finally, $\Delta_{p}>0$ and $\Delta_{m}>0$ are (constant) design parameters, which can be tuned by bearing in mind that smaller values of $\Delta_{m}$ lead to increased observer jumps (and reduced peaking) of the hybrid observer dynamics, while large values of $\Delta_{m}$ induce less resets and possibly no resets at all for very large values.

Based on the above observation, given any hybrid solution $\xi$ to the hybrid closed-loop (1), (16), denoting $\left(t_{j}\right)$ the sequence in $\mathbb{R}$ such that $\left(t_{j}, j\right)$ and $\left(t_{j}, j+1\right) \in \operatorname{dom} \xi$, and any $(t, j) \in \operatorname{dom} \xi$ such that, for some $i \in\{1, \ldots, n\}$, $\eta_{i}\left(t_{j}, j\right)=0$ and $\zeta_{i}\left(t_{j}, j\right)=e_{p i}\left(t_{j}, j\right)$, we have the following property:

$$
\zeta_{i}(t, j)=e_{p i}^{2}\left(t_{j}, j\right), \eta_{i}(t, j)=\int_{t_{j}}^{t} e_{p i}^{2}(\tau, j) d \tau
$$

for all $i=1, \ldots, n$. The next lemma is the main motivation for the introduction of the extra states.

Lemma 2 Consider any solution $\xi$ to the hybrid closed loop between plant (1) and observer (16) starting from initial conditions satisfying $\eta(0,0)=0$ and $\zeta(0,0)=$ $e_{p}(0,0)$. For each $(t, j) \in \operatorname{dom} \xi$, if $\xi(t, j) \in \mathcal{D}_{i}^{m}$ for some $i \in\{1, \ldots, n\}$, then $e_{p i}(t, j) e_{v i}(t, j) \leq 0$.

The following simple claim will be useful for the proof of Lemma 2.

Claim 1 Given any two non-negative reals satisfying $0 \leq t_{j}<t$, a scalar $\delta \in \mathbb{R}$ and a continuous function $\varphi(s), s \in\left[t_{j}, t\right]$, assume that:

$$
\int_{t_{j}}^{t} \varphi(\tau) d \tau \leq \delta, \text { and } \delta \leq \int_{t_{j}}^{s} \varphi(\tau) d \tau, \forall s \in\left[t_{j}, t\right),
$$

then $\varphi(t) \leq 0$.

Proof. First note that $\int_{t_{j}}^{t} \varphi(\tau) d \tau=\lim _{s \rightarrow t} \int_{t_{j}}^{s} \varphi(\tau) d \tau$ and thus it follows from (18) that $\int_{t_{j}}^{t} \varphi(\tau) d \tau=\delta$. Moreover defining the continuously differentiable function $\Psi$ by $\Psi: s \in\left[t_{j}, t\right) \mapsto \int_{t_{j}}^{s} \varphi(\tau) d \tau$, it follows from the second inequality in (18) that $\Psi(t) \leq \Psi(s)$, for all $s$ in $\left[t_{j}, t\right)$. Considering the derivative of $\Psi$ at $t$, this inequality implies $\varphi(t) \leq 0$.

Proof of Lemma 2. For all $(t, j) \geq\left(t_{j}, j\right) \in \operatorname{dom} \xi$, $s \mapsto \xi(s, j)$ is absolutely continuous and in particular (4) makes sense for all almost all $s \in\left[t_{j}, t\right)$. By multiplying the first equation in (4) (with $i=k$ ) by $2 e_{p i}$, we get $2 e_{p i} \dot{e}_{p i}=2 \ell e_{p i} e_{v i}-2 \ell k_{1} e_{p i}^{2}$, which can be integrated between $t_{j}$ and $s \in\left[t_{j}, t\right]$ to get

$$
\begin{aligned}
e_{p i}^{2}(s, j)- & e_{p i}^{2}\left(t_{j}, j\right)=\int_{t_{j}}^{s} \frac{d}{d \tau} e_{p i}^{2}(\tau, j) d \tau \\
& =\int_{t_{j}}^{s} \varphi(\tau) d \tau-2 \ell k_{1} \int_{t_{j}}^{s} e_{p i}^{2}(\tau, j) d \tau
\end{aligned}
$$

where $\varphi(\tau)=2 \ell e_{p i}(\tau, j) e_{v i}(\tau, j)$. Using the identities in (17) (which hold for $j \geq 1$ ), this implies:

$$
\int_{t_{j}}^{s} \varphi(\tau) d \tau=e_{p i}^{2}(s, j)-\zeta_{i}(s, j)+2 \ell k_{1} \eta_{i}(s, j) .
$$

Since we also have $\xi(s, j) \in \mathcal{C}$ for all $s \in\left[t_{j}, t\right)$ (otherwise the solution could not flow in this interval), then using the definition of flow set in (16d) and the assumption of the lemma, we have that

$$
\begin{aligned}
& e_{p i}^{2}(s, j)-\zeta_{i}(s, j)+2 \ell k_{1} \eta_{i}(s, j) \geq-\Delta_{m}, \quad \forall s \in\left[t_{j}, t\right) \\
& e_{p i}^{2}(t, j)-\zeta_{i}(t, j)+2 \ell k_{1} \eta_{i}(t, j) \leq-\Delta_{m}
\end{aligned}
$$

which, combined with (19), implies equation (18) with $\delta=-\Delta_{m}$ and then by Claim 1 we have $\varphi(t)=2 \ell e_{p i}(t, j) e_{v i}(t, j) \leq 0$, which completes the proof. 
In light of Lemma 2, the rationale behind the hybrid observer dynamics (16) can now be explained. As long as the solution $\xi$ belongs to the flow set, the observer dynamics coincides with that of the continuous-time highgain observer. However, the extra variables $\zeta$ and $\eta$ keep track of the quantities in (17) for each $i \in\{1, \ldots, n\}$. These quantities are used in the jump/flow rules of (16d) to detect (based on Lemma 2) whether a substate $e_{i}$ belongs to the second/fourth quadrant. In particular, once we detect $\xi \in \mathcal{D}_{i}^{m}$ for some $i$, then from the last equation in (16d) we have $e_{p i}^{2}-\zeta_{i}+2 \ell k_{1} \xi_{i} \leq-\Delta_{m}$ and Lemma 2 applies. Such an event triggers the jump rule in (16c) which implies that $e_{i}^{+}=\left(-\alpha e_{p i}, e_{v i}\right)$ and then non-increase of the Lyapunov function follows from Lemma 1. This result is formalized in the next statement which is the main result of this paper.

Theorem 1 Consider plant (1) satisfying (2), and observer (16). Assume that (6) holds for some $P$ and $\vartheta$. Then there exist positive scalars $M, \lambda$ such that the estimation error $e=x-\hat{x}$ satisfies

$$
|e(t, j)| \leq M \exp (-\lambda t)|e(0,0)|, \quad \forall(t, j) \in \operatorname{dom} e,
$$

for any solution $\xi$ to the closed loop between plant (1) and observer (16) starting from initial conditions satisfying $\eta(0,0)=0$ and $\zeta(0,0)=e_{p}(0,0)$.

Remark 6 Due to the expressions of the flow set, it yields that $(x, \hat{x}, \zeta, \eta)$ is in $\mathcal{C}$ if and only if

$$
\frac{-\Delta_{m}-e_{p i}^{2}+\zeta_{i}}{2 \ell k_{i}} \leq \eta_{i} \leq \frac{\Delta_{p}-e_{p i}^{2}+\zeta_{i}}{2 \ell k_{i}}, \forall i .
$$

In light of Proposition 2 (ensuring that all maximal solutions have unbounded domain in the ordinary time direction $t$ ), Theorem 1 states the exponential convergence to zero of $e_{p i}$. Since along flow $\zeta_{i}$ are constant, this implies that $\eta_{i}$ are also bounded along flow. With the expression of the jump map in (16a)-(16c), it yields boundedness of the variables $\zeta$ and $\eta$ along the solutions to the hybrid system.

Proof of Theorem 1. Consider the Lyapunov function $V(e)=e^{T}\left(P \otimes I_{n}\right) e$ as in $(7)$, which satisfies:

$a_{1}|e|^{2} \leq V(e) \leq a_{2}|e|^{2}, \quad \forall e \in \mathbb{R}^{2 n}$,

for some positive scalars $a_{1}, a_{2}$. Moreover, since the flow dynamics in (16a) coincides with the continuous-time high-gain observer dynamics, one has from Proposition 6 (see in particular (11) and the scalar $\epsilon_{V}$ therein introduced):

$\dot{V}(\xi) \leq-\epsilon_{V}|e|^{2} \leq-\frac{\epsilon_{V}}{a_{2}} V(e), \quad \forall \xi \in \mathcal{C}$

Regarding the change of $V$ across jumps, notice that from $(16 \mathrm{~b}) \xi \in \mathcal{D}_{i}^{p}$ leads to $e^{+}=x^{+}-\hat{x}^{+}=x-\hat{x}=$ $e$, which means $V\left(e^{+}\right)-V(e)=0, \forall \xi \in \mathcal{D}_{i}^{p}$. As for $\mathcal{D}_{i}^{m}$ we need to specifically look into solutions and apply Lemmas 1 and 2. In particular, for any solution $\xi(t, j)$, $(t, j) \in \operatorname{dom} \xi$, if $\xi\left(t_{j}, j-1\right) \in \mathcal{D}_{i}^{m}$ for some jump time $t_{j}$, then we have from Lemma 2 that $e_{p i}\left(t_{j}, j-1\right) e_{v i}\left(t_{j}, j-\right.$ $1) \leq 0$, which, from Lemma 1 implies $V\left(e^{+}\left(t_{j}, j-1\right)\right)=$ $V\left(\bar{e}\left(t_{j}, j\right)\right) \leq V\left(e^{+}\left(t_{j}, j-1\right)\right)$. More specifically, we have for all $\left(t_{h}, \bar{h}\right) \in \operatorname{dom} \xi$,

$V\left(e\left(t_{h}, h\right)\right)-V\left(e\left(t_{h}, h-1\right)\right) \leq 0$.

Consider now a hybrid time $(t, j) \in \operatorname{dom} \xi$. and, for simplicity, let us denote $t_{j+1}=t$ (even though $t_{j+1}$ is not necessarily a jump time). Along any flowing interval $\left[t_{h}, t_{h+1}\right], h \in\{0, \ldots, j\}$, we know that the solution belongs to $\mathcal{C}$ (because $\mathcal{C}$ is closed) and then we may use (22) to characterize the continuous variation of $V$ as follows:

$$
\frac{d}{d \tau} V(e(\tau, h)) \leq-\frac{\epsilon_{V}}{a_{2}} V(e(\tau, h)), \forall \tau \in\left(t_{h}, t_{h+1}\right)
$$

Using standard comparison theory and the fact that $V$ is continuous along flow, we may transform (24) into an exponential bound. Then, concatenating all the bounds from $h=j$ to $h=0$ and exploiting recursively (23), we get:

$$
\begin{aligned}
V(e(t, j)) & \leq \exp \left(-\frac{\epsilon_{V}}{a_{2}}\left(t-t_{j}\right)\right) V\left(e\left(t_{j}, j\right)\right) \\
& \leq \exp \left(-\frac{\epsilon_{V}}{a_{2}}\left(t-t_{j}\right)\right) V\left(e\left(t_{j}, j-1\right)\right) \leq \ldots \leq \\
& \leq \exp \left(-\frac{\epsilon_{V}}{a_{2}}\left(t-t_{0}\right)\right) V\left(e\left(t_{0}, 0\right)\right)
\end{aligned}
$$

where $t_{0}=0$ by definition. Finally, using (21) we get

$$
\begin{aligned}
|e(t, j)|^{2} & \leq \frac{1}{a_{1}} V(e(t, j)) \leq \frac{1}{a_{1}} \exp \left(-\frac{\epsilon_{V}}{a_{2}} t\right) V(e(0,0)) \\
& \leq \frac{a_{2}}{a_{1}} \exp \left(-\frac{\epsilon_{V}}{a_{2}} t\right)|e(0,0)|^{2}
\end{aligned}
$$

completing the proof with $\lambda=\frac{\epsilon_{V}}{2 a_{2}}$ and $M=\sqrt{\frac{a_{2}}{a_{1}}}$.

Proof of Proposition 2. To show that all (maximal) solutions are complete, let us first note that the Viability Condition (VC) of (Goebel et al., 2012, Prop 6.10) holds and that $\mathcal{C} \cup \mathcal{D}=\mathbb{R}^{6 n}$. Moreover, using (22) and (23), we obtain that function $V$ is nonincreasing along solutions, therefore solutions cannot escape to infinity in finite time. Therefore, with (Goebel et al. 2012, Prop $6.10)$, all maximal solutions are complete. Moreover it follows from (25) that

$|e(t, j)| \leq \sqrt{\frac{a_{2}}{a_{1}}}|e(0,0)|, \quad \forall(t, j) \in \operatorname{dom} e$. 
Then, using (4) and (5), we get for almost every $t$ and for all $j$ such that $(t, j) \in \operatorname{dom} e,|\dot{e}(t, j)| \leq$ $\left(\ell\left|A_{e} \otimes I_{n}\right| \sqrt{\frac{a_{2}}{a_{1}}}+n L_{\delta}\right)|e(0,0)|=: M_{e}|e(0,0)|$. Based on the definition of jump set in $(16 \mathrm{~d})$, we know that the solution does not jump as long as $\theta_{i}=\left|e_{p i}^{2}-\zeta_{i}+2 \ell k_{1} \eta_{i}\right|<$ $\min \left\{\Delta_{p}, \Delta_{m}\right\}=: \delta$, for all $i=1, \ldots, n$. This boundedness property, together with the fact that $\mathcal{C} \cup \mathcal{D}$ spans the whole space, implies that solutions can always be continued and then maximal solutions are complete.

Consider now identities (17) and conclude that immediately after each jump time $t_{j}$ with the jump triggered by $\mathcal{D}_{i}^{p} \cup \mathcal{D}_{i}^{m}$, we have $\theta_{i}\left(t_{j}, j\right)=0$. Moreover, using (17) and $(26)$ we have

$|\dot{\theta}| \leq 4|\dot{e}||e|+2 \ell k_{1}|e|^{2} \leq\left(4 M_{e} \sqrt{\frac{a_{2}}{a_{1}}}+2 \ell k_{1} \frac{a_{2}}{a_{1}}\right)|e(0,0)|^{2}=:$ $M_{\theta}$.

Therefore, for each $t \in\left[t_{j}, t_{j}+\delta / M_{\theta}\right)$, such that $(t, h) \in$ dom $e$ for some $h \geq j$, we have $\theta_{i}(t, h) \leq \delta$, which implies by definition of $\delta$ that $\xi(t, h) \notin \mathcal{D}_{i}^{p} \cup \mathcal{D}_{i}^{m}$. Therefore any pair of hybrid times such that the state is in the same jump subset $\mathcal{D}_{i}^{p} \cup \mathcal{D}_{i}^{m}$ should be separated by at least $\delta / M_{\theta}$ flowing time, for each $i \in\{1, \ldots, n\}$. Since there are $n$ instances of subsets $\mathcal{D}_{i}^{p} \cup \mathcal{D}_{i}^{m}$, we conclude that each solution jumps at most $n$ times in any ordinary time interval of length $\delta / M_{\theta}$. In other words, following the characterization of (Cai et al., 2008, Prop. 1.1) all solutions to the hybrid observer closed loop satisfy an average dwell-time condition with parameters $\left(M_{\theta} / \delta, n\right)$.

Example 2 Let us reconsider Example 1 and compare the classical high-gain solution with the hybrid solution having the same parameters and with the selection $\Delta_{p}=$ $\Delta_{m}=0.01$ and $\alpha=0.5$. Starting from the same initial condition as in Example 1, we obtain the phase portraits of $\left(e_{1}, e_{2}\right)$ and $\left(x_{1}-\hat{x}_{1}, x_{2}-\hat{x}_{2}\right)$ given in Figure 2.

By comparing Figures 1 and 2, we note that the highgain reduces dramatically the peaking experienced in the linear case. The Lyapunov function $V(e)=e^{T} P e$ decreases exponentially fast to zero when flowing, while it does not increase across jumps, as established, respectively, in (22) and (23) in the proof of Theorem 1.

In particular, Figure 3 shows the ordinary time evolution of this Lyapunov function for various selections of $\alpha$ within the allowable range. From the top plot it is apparent that smaller values of $\alpha$ lead to a more substantial decrease of the Lyapunov function across jumps (despite the fact that only non-increase is guaranteed by our results). On the other hand, comparable transients are experienced on the norm squared of the unscaled error $\left(x_{1}-\hat{x}_{1}, x_{2}-\hat{x}_{2}\right)$ (bottom plot), thus showing that, regardless of the selection of $\alpha$, the hybrid high-gain ob-
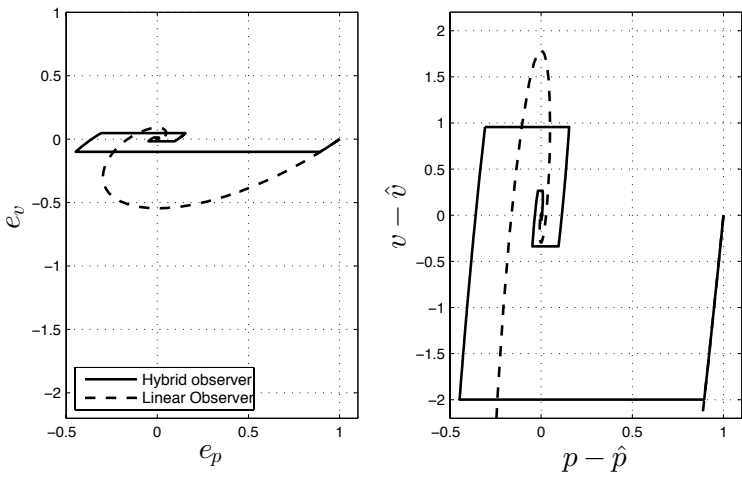

Figure 2. Phase portrait of the coordinates $\left(e_{1}, e_{2}\right)$ (left) and of $\left(x_{1}-\hat{x}_{1}, x_{2}-\hat{x}_{2}\right)$ (right), with $\alpha=0.5$.
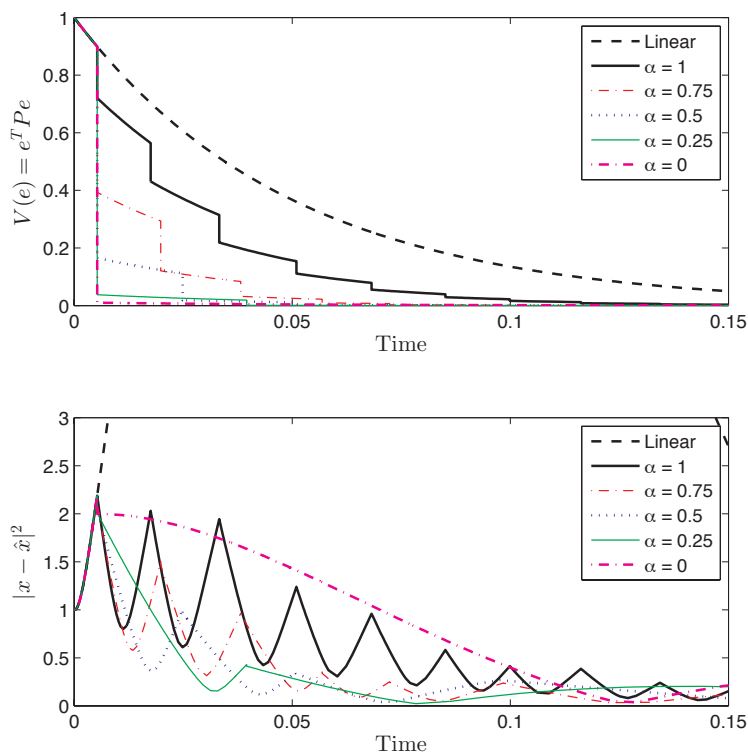

Figure 3. Time-evolution of the Lyapunov function $V(e)$ (top) and of the squared norm of the non-scaled error (bottom), with various selections of $\alpha$.

server shows a tremendous improvement on the peaking of the standard high-gain solution (the dashed curve in the lower plot reaches a peak value of 11.9). For this specific simulation, the selection $\alpha=0.25$ appears to be the most desirable one, and the selection $\alpha=0$ surprisingly leads to just a few resets.

Remark 7 As illustrated in Example 2 below, the hybrid high-gain observer proposed here is capable of suppressing the peaking behavior, as illustrated in Figure 3, where the estimation error $|x-\hat{x}|$ is represented. When $\Delta_{p}$ and $\Delta_{m}$ are selected sufficiently small as compared to the period of the revolving trajectories, once the error enters the "peaking-prone" region $e_{p i} e_{v i} \leq 0$, the resetting rule in (16c) changes the sign of $e_{p i}$, thereby transferring the trajectory to the good region $e_{p i} e_{v i} \geq 0$ where the error is decreasing again. Since the sign of $e_{p i} e_{v i}$ is detected by our algorithm based on an integral 
inequality, then some increase of the norm of the error should be expected (see e.g., the black curves in the bottom plot of Figure 3) but this increase is expected to be significantly smaller than the one experienced from peaking (the dashed curve in the bottom plot of Figure 3 reaches a peak of 11.9).

\section{Application to a planar robot example}

A relevant class of systems that falls into the setting of equation (1) is that of fully actuated Euler-Lagrange systems (see, e.g., Ortega et al. (1998); Sciavicco and Siciliano (2000)). Denoting by $q$ the generalized positions and by $\dot{q} \in \mathbb{R}^{2}$ the generalized velocities, the equations of motion of these systems can be compactly represented as (see, e.g., (Sciavicco and Siciliano, 2000, §7.1))

$$
D(q) \ddot{q}+C(q, \dot{q}) \dot{q}+h(q)=u
$$

where $D(q)$ is the inertia matrix, which satisfies $d_{\text {min }} I \leq$ $D(q) \leq d_{\max } I$ for all $q$ and for a suitable selection of positive values $d_{\min }, d_{\max }, C(q, \dot{q}) \dot{q}$ comprises centrifugal and Coriolis terms (namely quadratic terms in $\dot{q}$ ), $h(q)$ is the gravitational vector, and $u$ represents the external forces/torques applied on each degree of freedom of the system.

Selecting $y=p=q$ and $v=\dot{q}$, model (27) can be represented as in (1) with selection $\psi(y)=0$ and

$\phi((p, v), u)=D^{-1}(q)(u-C(p, v) v-h(p))$.

While we may apply our technique to any such EulerLagrange system, we concentrate on a planar two-link rigid robot manipulator with rotational joints, used in many existing works (see, e.g., (Sciavicco and Siciliano, 2000, §7.3.2)). We use the parameters used in Morabito et al. (2004).

A typical control selection for robotic systems is the feedback linearizing controller, which can be implemented as follows in feedback from the measured position and the observed velocity:

$u=C(p, v) v+h(p)-D(p)\left(K_{p} p+K_{d} v\right)$,

where we select $K_{p}=\left[\begin{array}{cc}20 & 0 \\ 0 & 20\end{array}\right]$ and $K_{d}=\left[\begin{array}{cc}20 & 0 \\ 0 & 10\end{array}\right]$ to induce a desirable and graceful response of the closed-loop system via state feedback (see the top curves in Figure 4). To describe a practical scenario, we carry out simulations by taking into account a disturbance acting on the position measurement $p$, corresponding to the presence of a quantizer, possibly arising from the use of incremental encoders that measure the joint positions. The quantizer level is chosen as $2 \pi / 3600$, namely we assume that the encoder has 10 steps per degree. Since it is known that high-gain observers are sensitive to measurement noise, in addition to the quantization level, we add a band limited white noise to the position measurements (after the quantization) having sample time 0.01 and noise power $10^{-7}$, so that the sensitivity to noise is comparatively assessed. 4 As customary in robotic applications, rather than relying on the conservative bound on $\ell$ arising from Proposition 1, we tune parameter $\ell$ with the goal of obtaining a suitable filtering action for the quantization noise, thereby obtaining the value $\ell=5$. We still implement the reduced order observer described in Remark 3 by computing a matrix $P_{e}$ from (6) by maximizing the allowable $L_{\delta}$ for this specific value of $\ell$ (similar to Remark 4 , this is a generalized eigenvalue problem). We also select $\Delta_{p}=\Delta_{m}=1 e-3$ so that resets are suitably induced during the transient response of the hybrid observer.

Figure 4 shows the simulation results starting from the initial condition $p(0)=(50,0)$ degrees and $v(0)=(50,50)$ degrees/sec. The peaking occurring with the bare high-gain solution is quite evident from the left graph on the third row, while this peaking is removed by both the hybrid high-gain solution and the reduced order solution of the second and fourth rows. Interestingly, most resets of the hybrid observer occur during the initial transient (when peaking must be removed) and only a few resets occur in the remaining part of the response (see the last row of the figure). This reveals that after the initial transient, the hybrid solution essentially produces responses coinciding with those of the high-gain solution (essentially without resets, except for seldom ones caused by noise). The advantage of the hybrid solution versus the reduced order one is evident at the steady state (right graphs in Figure 4), where unpleasant bursts can be inspected in the curves of the second row, corresponding to the reduced order solution. These bursts arise from the fact that the high-gain and hybrid high-gain solutions are both strictly proper observers, thereby providing a filtering action that is impossible to obtain from the reduced order approach, where the position measurement is directly fed to the input of the controller. 5 No disturbance is used in the state feedback simulation (upper curves) to better illustrate the desirable features of the (ideal) undisturbed state feedback response. Note also that in the presence of noise having larger amplitude, one expects a graceful performance degradation of the proposed scheme due to the semiglobal practical stability results proven in Goebel et al. 2012, Thms $7.20 \& 7.21$ ), which hold for our solution because it satisfies the hybrid basic conditions by

\footnotetext{
4 We realize that the presence of noise after a quantizer is not physically meaningful but we expressively carry out this test to verify the observers responses in a worst case scenario. 5 Maybe the bursts, obtained with the reduced order observer, may be avoided by adding an appropriate one-order filter to recover the same dimension of the dynamics as the the hybrid observer. However the tuning of this filter may be problematic.
} 

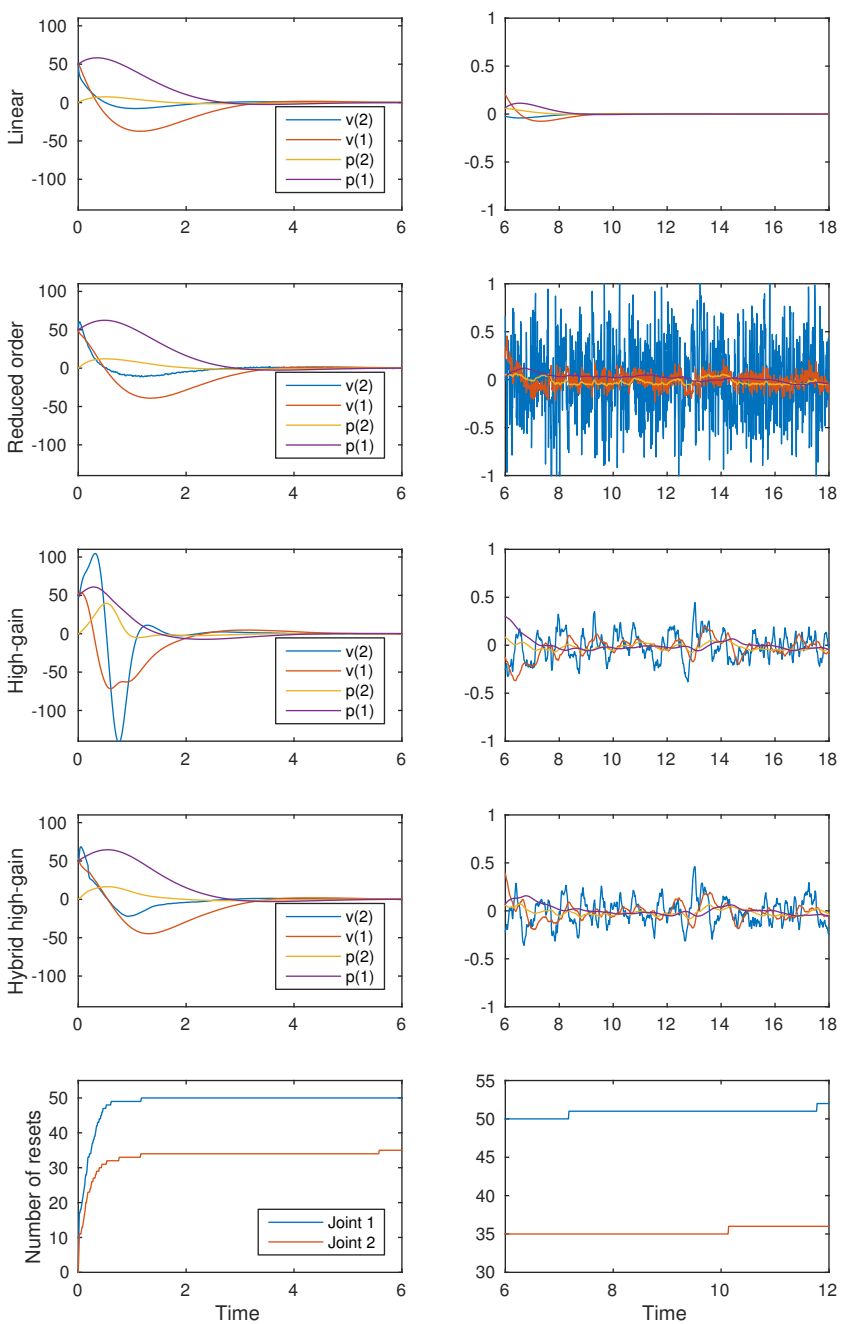

Figure 4. Output responses for the two link robot arm: state feedback (top), classical high-gain (middle-top), hybrid high-gain (middle-bottom), Remark 3 (bottom).

virtue of the regularity of the data in our solution.

\section{Conclusion}

Considering a family of nonlinearly interconnected second order plants, the high-gain observer design has been revised within a hybrid dynamical systems framework, with the objective to avoid the peaking phenomenon. A hybrid high-gain observer has been proposed by augmenting the original continuous-time high-gain observer dynamics with a suitable resetting rule relying on the observer state. This approach has been illustrated on an example borrowed from the literature, and also by means of simulations on a planar robot. The hybrid high-gain observer is also interesting when compared to a reduced order observer, since both observers reduce peaking, but the hybrid high-gain observer exhibits a few number of jumps and avoids bursts of the input, that are present when closing the loop with a reduced order observer.

\section{References}

Ailon, A., Ortega, R., 1993. An observer-based set-point controller for robot manipulators with flexible joints. Systems \& Control Letters 21 (4), 329-335.

Andrieu, V., Praly, L., 2006. On the existence of KazantzisKravaris / Luenberger Observers. SIAM Journal on Control and Optimization 45 (2), 432-456.

Andrieu, V., Praly, L., Astolfi, A., 2009. High gain observers with updated gain and homogeneous correction terms. Automatica 45 (2), 422-428.

Arcak, M., Kokotovic, P., 2001. Observer-based control of systems with slope-restricted nonlinearities. IEEE Transactions on Automatic Control 46 (7), 1146-1150.

Astolfi, D., Praly, L., Dec 2013. Output feedback stabilization for SISO nonlinear systems with an observer in the original coordinates. In: Conference on Decision and Control. Florence, Italy, pp. 5927-5932.

Besançon, G., 2000. Remarks on nonlinear adaptive observer design. Systems and Control Letters 41 (4), 271-280.

Besançon, G., 2007. Nonlinear observers and applications. Vol. 363. Springer Verlag.

Cai, C., Teel, A., Goebel, R., 2008. Smooth Lyapunov functions for hybrid systems Part II:(pre) asymptotically stable compact sets. IEEE Transactions on Automatic Control 53 (3), 734-748.

Esfandiari, F., Khalil, H., 1992. Output feedback stabilization of fully linearizable systems. International Journal of Control 56 (5), 1007-1037.

Gauthier, J.-P., Hammouri, H., Othman, S., 1992. A simple observer for nonlinear systems applications to bioreactors. IEEE Transactions on Automatic Control 37 (6), 875-880.

Gauthier, J. P., Kupka, I., 2001. Deterministic observation theory and applications. Cambridge University Press.

Goebel, R., Sanfelice, R., Teel, A., 2009. Hybrid dynamical systems. IEEE Control Systems Magazine 29 (2), 28-93.

Goebel, R., Sanfelice, R., Teel, A., 2012. Hybrid Dynamical Systems: modeling, stability, and robustness. Princeton University Press.

Kaliora, G., Astolfi, A., Praly, L., 2006. Norm estimators and global output feedback stabilization of nonlinear systems WithISS inverse dynamics. IEEE Transactions on Automatic Control 51 (3), 493-498.

Khalil, H., 1999. High-gain observers in nonlinear feedback control. In: Nijmeijer, H., Fossen, T. (Eds.), New directions in nonlinear observer design. Springer, pp. 249-268.

Khalil, H., 2002. Nonlinear Systems, 3rd Edition. Prentice-Hall.

Khalil, H. K., Praly, L., 2013. High-gain observers in nonlinear feedback control. International Journal of Robust and Nonlinear Control 24 (6), 993-1015.

Kokotovic, P., 1992. The joy of feedback: nonlinear and adaptive. IEEE Control Systems Magazine 12 (3), 7-17.

Lei, H., Wei, J., Lin, W., Dec 2005. A global observer for observable autonomous systems with bounded solution trajectories. In: Decision and Control, 2005 and 2005 European Control Conference. CDC-ECC '05. 44th IEEE Conference on. pp. 1911-1916.

Morabito, F., Nicosia, S., Teel, A., Zaccarian, L., 2004. Measuring and improving performance in anti-windup laws for robot manipulators. In: Siciliano, B., Luca, A. D., Melchiorri, C., Casalino, G. (Eds.), Advances in Control of Articulated and Mobile Robots. Springer Tracts in Advanced Robotics, Ch. 3, pp. $61-85$.

Nicosia, S., Tomei, P., Tornambé, A., 1989. An approximate observer for a class of nonlinear systems. Systems \& Control Letters 13 (1), 43-51. 
Oliveira, T. R., Peixoto, A. J., Costa, R. R., Hsu, L., 2010. Dwelltime and disturbance monitoring for peaking avoidance and performance improvement in high-gain observer based sliding mode control. Dynamics of Continuous, Discrete and Impulsive Systems Series B: Applications and Algorithms 17 (6), 839874.

Oliveira, T. R., Peixoto, A. J., Hsu, L., 2013. Peaking free outputfeedback exact tracking of uncertain nonlinear systems via dwell-time and norm observers. International Journal of Robust and Nonlinear Control 23 (5), 483-513.

Ortega, R., Loria, A., Nicklasson, P., Sira-Ramirez, H., 1998. Passivity-Based Control of Euler-Lagrange Systems. Communications and control engineering. Springer.

Paesa, D., Franco, C., Llorente, S., Lopez-Nicolas, G., Saguez, C., 2012. Reset adaptive observer for a class of nonlinear systems. IEEE Transactions on Automatic Control 57 (2), 506-511.

Phillips, S., Sanfelice, R. G., 2015. Robust asymptotic stability of desynchronization in impulse-coupled oscillators. IEEE Transactions on Control of Network Systems (to appear).

Prieur, C., Tarbouriech, S., Zaccarian, L., Dec. 2012. Hybrid highgain observers without peaking for planar nonlinear systems. In: Conference on Decision and Control. Maui (HI), USA, pp. 6175-6180.

Raff, T., Allgöwer, F., 2008. An observer that converges in finite time due to measurement-based state updates. In: IFAC World Congress. Seoul, Korea, pp. 2693-2695.

Rockafellar, R., Wets, R., 1998. Variational Analysis. Springer.

Sanfelice, R., May 2014. Input-output-to-state stability tools for hybrid systems and their interconnections. IEEE Transactions on Automatic Control 59 (5), 1360-1366.

Sciavicco, L., Siciliano, B., 2000. Modelling and control of robot manipulators. Springer.

Sepulchre, R., Janković, M., Kokotović, P. V., 1997. Constructive nonlinear control. Communications and Control Engineering Series. Springer-Verlag.

Teel, A., Praly, L., 1994. Global stabilizability and observability imply semi-global stabilizability by output feedback. Systems \& Control Letters 22 (5), 313-325. 